\title{
Intravascular Ultrasound-Based Imaging of Vasa Vasorum for the Detection of Vulnerable Atherosclerotic Plaque
}

\author{
Sean M. O'Malley ${ }^{1}$, Manolis Vavuranakis ${ }^{2}$, \\ Morteza Naghavi ${ }^{3}$, and Ioannis A. Kakadiaris ${ }^{1}$ \\ 1 Visual Computing Lab, Dept. of Computer Science, Univ. of Houston \\ 2 Dept. of Cardiology, Univ. of Athens \\ 3 Association for Eradication of Heart Attack
}

\begin{abstract}
Vulnerable plaques are dangerous atherosclerotic lesions that bear a high risk of complications that can lead to heart attacks and strokes. These plaques are known to be chronically inflamed. The vasa vasorum (VV) are microvessels that nourish vessel walls. Proliferation of $\mathrm{VV}$ is part of the "response to injury" phenomenon in the process of plaque formation. Recent evidence has shown strong correlations between neovessel formation and macrophage infiltration in atherosclerotic plaque, suggesting VV density as a surrogate marker of plaque inflammation and vulnerability. We have developed a novel method for imaging and analyzing the density and perfusion of VV in human coronary atherosclerotic plaques using intravascular ultrasound (IVUS). Images are taken during the injection of a microbubble contrast agent and the spatiotemporal changes of the IVUS signal are monitored using enhancement-detection techniques. We present analyses of in vivo human coronary cases that, for the first time, demonstrate the feasibility of IVUS imaging of VV.
\end{abstract}

\section{Introduction}

Vulnerable plaques are subsets of atherosclerotic lesions that rupture and create blood clots resulting in acute coronary syndrome and sudden cardiac death. Plaque inflammation plays a central role in its vulnerability to future complications (e.g., rupture, hemorrhage, distal emboli, and acute stenosis). The search for an intracoronary technology capable of imaging both plaque morphology and activity (inflammation) is currently a very active topic in the cardiology community [1]. Despite major advances in the development of other intravascular imaging techniques, intravascular ultrasound (IVUS) remains the most widely-available technology to interventional cardiologists. However, the major drawback of IVUS has been its inability to gauge plaque inflammation.

The vasa vasorum (VV) are microvessels that nourish vessel walls (Fig. 1(a)]. In conditions with extensive neovessel formations such as atherosclerotic plaques, tumor angiogenesis, and diabetic retinopathy, most are fragile and prone to leak or rupture. In the field of atherosclerosis, recent evidence indicates that 


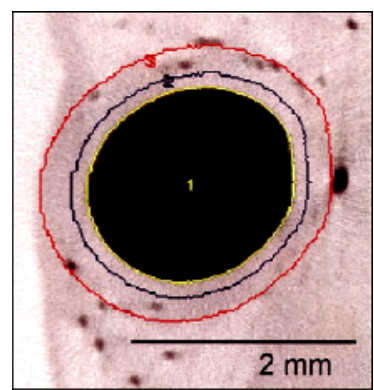

(a)

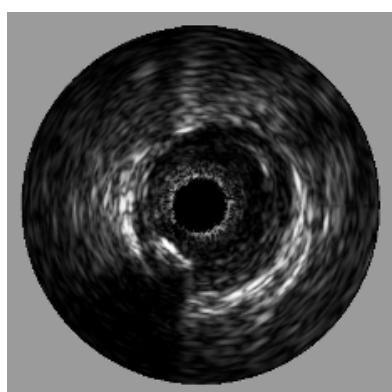

(b)

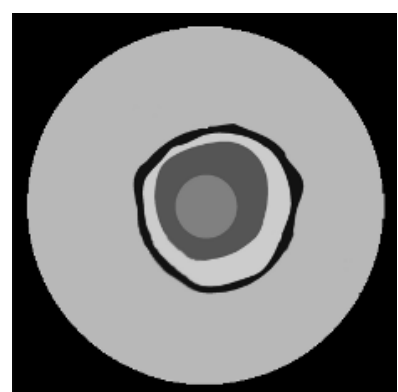

(c)

Fig. 1. (a) Vasa vasorum histology (reprinted from [9]) in relation to (b) a typical IVUS frame and (c) its labeled regions (from the center outward: the catheter; the echofree lumen; the plaque area with the intima, its membranous leading edge; the echofree media; and the adventitia)

proliferation of $\mathrm{VV}$ is a preceding or concomitant factor associated with plaque inflammation and instability [2, 3].

We have developed a novel method which, for the first time, enables IVUS imaging of atherosclerotic plaque inflammation based on quantification of $\mathrm{VV}$ density and perfusion. Our primary contributions are: 1) an IVUS acquisition protocol which utilizes a recently-developed ultrasound contrast agent to induce echogenicity in the VV, and 2) an automated algorithm for the detection, quantification, and visualization of VV in the resulting contrast-enhanced image sequences.

Related work includes plaque characterization [4, 5] and the imaging of myocardial perfusion [6] and angiogenesis [7]. Recent research into IVUS imaging has been primarily aimed at automating the segmentation task for the major IVUS features [8] (Figs. [1](b) (c)]. To the best of our knowledge, there have been no previous reports of automated detection and imaging of $\mathrm{VV}$.

\section{Materials and Methods}

IVUS System: We use both a solid-state phased-array $20 \mathrm{MHz}$ scanner (Volcano Therapeutics Inc. - Invision ${ }^{\mathrm{TM}}$ ) and a rotating single-crystal $40 \mathrm{MHz}$ scanner (Boston Scientific Inc. - Galaxy ${ }^{\mathrm{TM}}$ ).

Microbubbles: We use Optison ${ }^{\mathrm{TM}}$, a new ultrasound contrast agent composed of albumin microspheres filled with octafluoropropane gas.

VV Imaging Protocol: 1) Acquire IVUS frames for several minutes to obtain unenhanced signal ("pre-injection" period); 2) inject contrast agent, temporarily washing out the frames due to luminal saturation ("during-injection" period); and 3 ) acquire frames for several minutes further to obtain signal indicating potentially enhanced areas due to perfusion into the VV ("post-injection" period). 
During all periods, the IVUS catheter is held fixed; 1 min into the final period, 5 cc normal saline is injected to flush out remaining microbubbles.

Imaging Vasa Vasorum Density: Following acquisition, our VV detection, quantification, and visualization method consists of three steps.

- Step 1: Track cardiac motion throughout the sequence to allow compensation for relative catheter/vessel movement (Sect. 2.1).

- Step 2: Perform enhancement detection using difference-imaging and statistical techniques (Sect. 2.2).

- Step 3: Quantify and visualize the resulting enhancement (Sect. 2.3).

\subsection{Step 1: Motion Compensation}

In stationary-catheter IVUS studies, maintaining a fixed catheter position with regard to an anatomic point of reference is impossible in practice due to the periodic motion of the heart. Relative motion between the catheter and vessel produces image sequences in which each frame deviates from its predecessors; this deviation makes analysis of a specific anatomic region-of-interest (ROI) difficult. Our studies were performed on IVUS sequences which lacked associated electrocardiogram gating data. Consequently, to track cardiac motion, we have developed a method inspired by Zhu et al. [10, in which cardiac phase is derived from the IVUS sequence itself. This allows us, in subsequent processing, to select frames according to their position in phase (and, by extension, their physical orientation). Phase extraction is accomplished through ROI selection, intensity metasignal generation, and filtering and signal reconstruction, as follows.

ROI Selection: We select a fixed ROI in the IVUS frame to monitor the changes in intensity in this region over time. We do not analyze data from the entire frame because the lumen/catheter region has little useful signal and the adventitial region has a very low signal-to-noise ratio. Instead, the intensity study is concentrated on the region between the luminal border and the adventitia. The ROI need not be a perfect segmentation of this region, however; we may simply compute an average frame over a particular time range (e.g., one cardiac period) and create a mask which on average contains the entire ROI 1

Intensity Metasignal Generation: To produce these metadata, we use one of two techniques: average intensity or inter-frame difference. The average intensity $g$ at frame $i$ over our ROI is given by $g_{i}=\frac{1}{n} \sum_{(x, y) \in \mathrm{ROI}} F_{i}(x, y)$, where $F$ is an IVUS frame. The inter-frame difference $d$ between frame $i$ and the previous frame $i-1$ is given by $d_{i}=\frac{1}{n} \sum_{(x, y) \in \mathrm{ROI}}\left|F_{i}(x, y)-F_{i-1}(x, y)\right|$ (in both cases, $n$ is the area in pixels of the ROI). Here we use these techniques interchangeably; typically the only difference in the methods is a phase shift between the resulting signals.

Filtering $\&$ Signal Reconstruction: Due to the spurious extrema present in the signal (Fig. 2(a)), it is necessary to apply a filter to isolate those maxima and

\footnotetext{
${ }^{1}$ As we rely on a fixed ROI here, we do not employ a segmentation algorithm.
} 


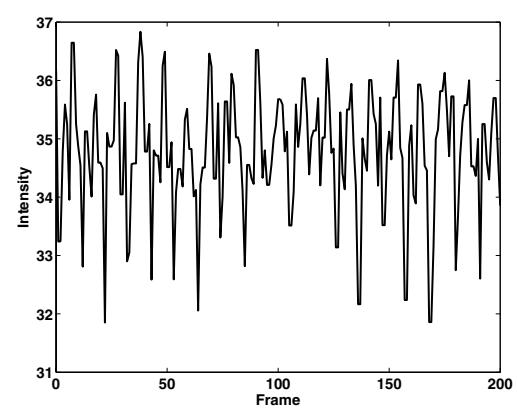

(a)

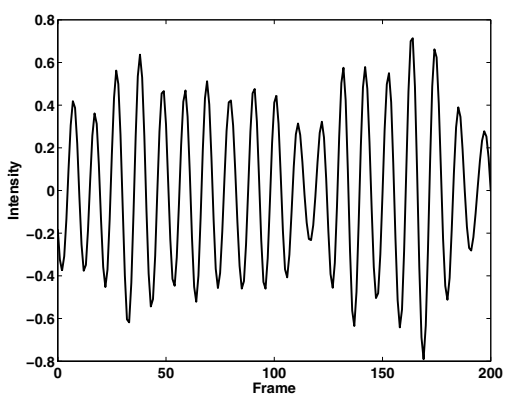

(b)

Fig. 2. (a) Plot of $g_{i}$ for the first 201 frames of Case 1 and (b) the filtered result

minima which represent the motion we are interested in. A Butterworth bandpass filter is applied, $H(\omega)=1 / \sqrt{1+\left[2\left(\omega-\omega_{c}\right) / \Delta \omega\right]^{2 m}}$, where the filter frequency $\omega_{c}$ is centered at the dominant cardiac frequency, the width $\Delta \omega=0.6\left(\omega_{c}\right)$, and the order $m=4$. We have found stationary-catheter IVUS studies to be robust with regard to automatic cardiac phase extraction as signals produced with this method exhibit a prominent peak in the frequency domain; this makes algorithmic determination of $\omega_{c}$ much more feasible than in previous pullback studies. While this peak is generally obvious to the human viewer, we have noted two other common sources of low-frequency noise which must be appropriately ignored: these include a prominent DC component related to the mean intensity or inter-frame difference, and other low-frequency components due to the microbubble washout during acquisition, which causes a varying mean grey-level to occur in the ROI over time. Once the signal is collected, transformed to the Fourier domain, $\omega_{c}$ is determined, and the signal is filtered, it is returned to the time domain (Fig. 2(b) . The set of frames associated with, e.g., the peaks in this time-domain signal are considered correlated by our definition.

\subsection{Step 2: Enhancement Detection}

The goal of enhancement detection is to localize and quantify the subtle intensity changes in the IVUS image sequence due to the presence of perfused VV in the field of view of the IVUS sensor following microbubble injection. For detection to be reliable, it must be invariant both to motion and to the presence of ultrasound (US) speckle noise in the image. The former issue was discussed previously (Sect. 2.1) while, to address the latter, our enhancement detection method combines difference imaging with temporal averaging to attenuate speckle.

Difference imaging, broadly, involves a "before" image $\left(I_{b}\right)$ and an "after" image $\left(I_{a}\right)$. Here, $I_{b}$ is a baseline frame derived from a set of phase-correlated pre-injection frames which are pixel-wise averaged for noise reduction. That is, if we have a set of $k$ phase-correlated pre-injection frames $\mathbf{F} \equiv\left\{F_{1} \ldots F_{k}\right\}$, then $I_{b}=\frac{1}{k} \sum_{i=1}^{k} F_{i}$. The number of frames averaged to produce this image may be 
fairly large as we are interested only in highly temporally-coherent features of the sequence for this baseline. The image $I_{a}$ is either a single post-injection frame or an average of correlated post-injection frames. Single frames are useful if producing a video sequence, while temporal averaging of the post-injection set to produce $I_{a}$ will suppress speckle noise along with temporally incoherent enhanced regions (e.g., passing microbubbles).

Once $I_{b}$ (baseline) and $I_{a}$ (post-injection) images are available, a difference image is produced by pixel-wise subtraction, $I_{d}=I_{a}-I_{b}$, with negative values thresholded to zero. This raw difference image shows areas of true enhancement in addition to low-valued areas of false enhancement due to US artifacts. Consequently, further thresholding must be applied to reduce the noisy appearance of the image, but this is done conservatively to avoid suppressing relevant enhancement. In this context, we have developed an automatic thresholding technique inspired by classical expectation-maximization algorithms and the work of Bruzzone and Prieto [11. A grey-level threshold is determined for $I_{d}$ under the assumption that it is a Bayesian mixture of probabilities $p(X)=$ $p\left(X / v_{u}\right) P\left(v_{u}\right)+p\left(X / v_{e}\right) P\left(v_{e}\right)$ where $p(X)$ is the overall probability density function (PDF) of the difference image, $p\left(X / v_{u}\right)$ and $p\left(X / v_{e}\right)$ are the PDF's of the unenhanced $(u)$ and enhanced $(e)$ pixels respectively, and $P\left(v_{u}\right)$ and $P\left(v_{e}\right)$ are the a priori probabilities of these classes (unknown quantities are initially estimated from histogram statistics). Following global thresholding, a Markov modeling technique is applied to account for spatial relationships. This takes the strength of a point of detected enhancement into consideration along with its neighborhood to determine whether the enhancement is salient; this has the effect of reducing spot noise while refining the edges of enhanced regions. Note that for the purposes of estimating probabilities and enhancement levels, only the pixels in a ROI of the frame are taken into consideration: typically the intimo-medial region where we expect to find VV. As per Sect. 2.1, this ROI is fixed and need not perfectly segment the region.

Video sequences may be generated to show the changes in microbubble perfusion over time. In this case, the baseline $I_{b}$ remains as defined previously, while the comparison images are a sequence of running averages of phase-correlated post-injection frames. That is, if a sequence of $k$ correlated post-injection frames $\mathbf{C} \equiv\left\{C_{1} \ldots C_{k}\right\}$ is available and a $p$-frame running average is used (where $p$ is odd), an $h$-frame video sequence may be produced, where $h=k-p+1$ and the raw difference image for a particular frame $i \in[1, h]$ is $I_{d}=\left(1 / p \sum_{j=1}^{p} C_{i+j-1}\right)-I_{b}$. In addition, by binning the frames by cardiac phase into a convenient data structure, it is trivial to extend this method to utilize all post-injection frames by associating each frame in a sequence with its $p-1$ correlated neighbors.

\subsection{Step 3: Quantification and Visualization of Enhancement}

To quantify enhancement over time, a signal is produced composed of the average enhancement per enhanced pixel (AEPEP) of each frame. This is defined as follows. If $\mathbf{P} \equiv\left\{P_{1}, \ldots, P_{q}\right\}$ is the set of intensities of all pixels labeled "en- 
hanced" in the ROI of the difference image after thresholding and refinement, then the AEPEP value for the frame is given by $\varepsilon=\frac{1}{q} \sum_{p \in \mathbf{P}} p$. A useful feature of the AEPEP metric is that it remains relatively constant in spite of changes in the apparent area of the enhanced regions due to US distortion. Metrics utilizing the entire ROI were found to be too noisy as measures of enhancement. In addition, we fit an approximating spline to the resulting AEPEP signal to highlight trends for the human observer. Where results are presented as color-coded images, the unit of intensity is a percentage indicating an enhanced pixel's difference level divided by the maximum grey-level difference (255).

\section{Results}

We conducted microbubble contrast-enhanced IVUS imaging on seven patients with coronary artery disease using the ACES ${ }^{\mathrm{TM}}$ (Analysis of Contrast-Enhanced Sequences) software developed for this project. Due to space limitations, data collected from one typical case is presented in detail and six cases presented in summary 2

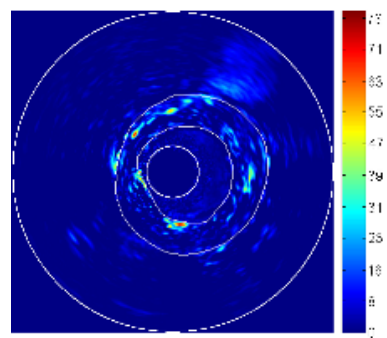

(a)

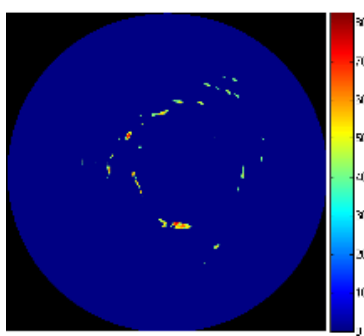

(b)

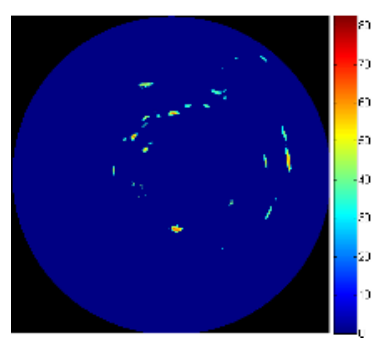

(c)

Fig. 3. Case 1: (a) raw difference image, and (b) (c) thresholded difference images at frames 600 and 800, respectively. Unit of intensity is \%, as discussed in Sect. 2.3

Case 1: This sequence consists of 1,073 frames sampled at 10 frames/s. Contrast agent (CA) first appears in the lumen in frame 404, complete washout due to lumen echo-opacity occurs from frames 452-490, and CA reaches a minimum in the lumen around frame 530. Figure 3(a) illustrates a raw difference image showing marked enhancement in the plaque region, particularly at 6 o'clock (note relation to calcified plaque in Fig. 4(a) and from 10-12 o'clock. Auto-thresholded difference images (Figs. 3)(b) (c) exhibit the changes in enhancement over time due to diminution. A magnification of an area of enhancement is shown in Fig. 4, this highlights the difficulty of imaging the echo-transparent VV under normal circumstances. Enhancement is plotted over time in Figs. 5(a) and 5(b) for the intimo-medial/plaque and adventitial regions respectively.

\footnotetext{
$\overline{{ }^{2}}$ Additional figures are available from http://www.vcl.uh.edu/CARDIA
} 


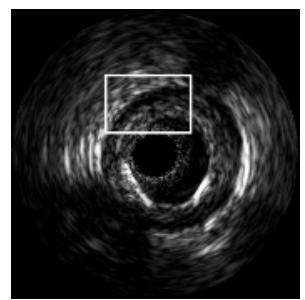

(a)

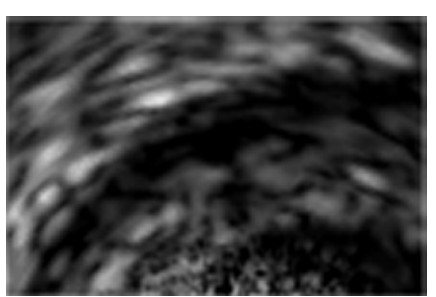

(b)

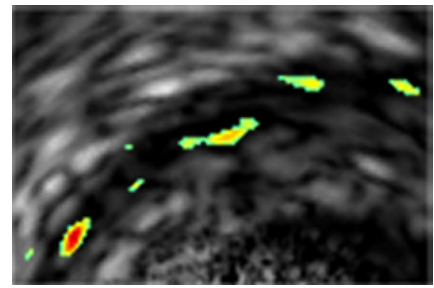

(c)

Fig. 4. (a) Unenhanced frame from Case 1, (b) a magnified ROI of the frame, and (c) the ROI with an overlay showing enhanced features invisible in (b). Observe conceptual agreement with histological observations reported in the literature (Fig. 1(a)].

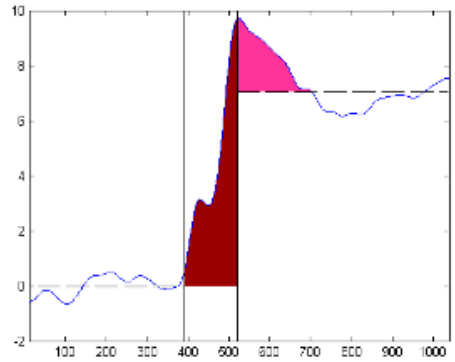

(a)

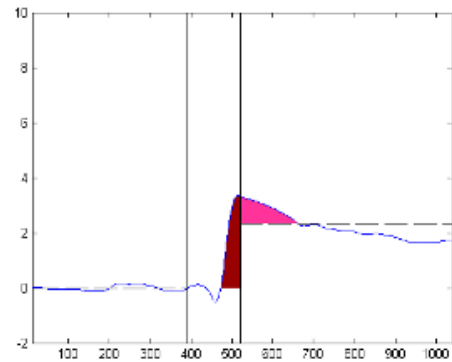

(b)

Fig. 5. Case 1: AEPEP over time for the (a) intimo-medial region and (b) adventitia. Pre- and post-injection means are shown (dotted horizontal lines; graph has been translated to $0 \%$ pre-injection mean). Injection start and peak are marked by vertical lines. Solid areas indicate the injection and return-to-baseline periods.

Summary: Enhancement statistics for six additional cases whose analyses were identical to that of Case 1 are presented in Table 11. The pre-injection mean AEPEP $\left(\mu_{\text {pre }}\right)$ scores report a positive value due to random speckle events occurring above each frame's enhancement threshold. Hence, the most meaningful

Table 1. Representative results from seven case studies.

\begin{tabular}{|l|l|l||r|}
\hline & $\mu_{\text {pre }}$ & $\mu_{\text {post }}$ & $R$ \\
\hline Case 1 & $2.41 \%$ & $9.13 \%$ & 3.79 \\
\hline Case 2 & $1.90 \%$ & $2.80 \%$ & 1.47 \\
\hline Case 3 & $1.53 \%$ & $3.97 \%$ & 2.59 \\
\hline Case 4 & $1.88 \%$ & $3.35 \%$ & 1.78 \\
\hline Case 5 & $1.48 \%$ & $2.27 \%$ & 1.53 \\
\hline Case 6 & $2.50 \%$ & $2.70 \%$ & 1.08 \\
\hline Case 7 & $4.00 \%$ & $5.50 \%$ & 1.37 \\
\hline
\end{tabular}


global enhancement metric we have found is the ratio of the post-injection mean $\left(\mu_{\text {post }}\right)$ to pre-injection mean, $R=\frac{\mu_{\text {post }}}{\mu_{p r e}}$. This ratio appears to correlate well with the strength and quantity of visible enhancement in our studies.

\section{Discussion}

We have described a novel method which, for the first time, enables IVUS imaging of VV presence. Due to the inherent limitations of in vivo human coronary IVUS studies, we were unable to correlate our findings with histopathological evidence of VV density. However, the significant changes in the IVUS signal after microbubble passage leave no doubt as to its ability to show contrast enhancement. Knowing that almost all blood perfusion in plaques comes through VV capillaries, we hypothesize that the enhancement is related to the density of $\mathrm{VV}$ in the vessel wall. A comparison of our images with the $\mu \mathrm{CT}$ and pathology images of VV reported in the literature [9] shows a conceptual agreement.

In vitro studies are currently underway to further gauge the accuracy and robustness of our technique. We are also investigating high-speed acquisition of the raw radiofrequency signal in order to more effectively measure the subtle changes in the backscatter from inside the plaque and adventitia. A clinical discussion of our results may be found in [12].

Acknowledgements. We would like to thank S. Carlier, C.J. Hartley, R.W. Metcalfe, N. Dib, R. Mehran, E. Falk, K. Gul, C. Stefanadis, T. Papaioannou, S. Vaina, M. Drakopoulou and I. Mitropoulos for valuable assistance. S. O'Malley is supported under a NSF Graduate Research Fellowship. This work supported in part by NSF Grant IIS-0431144. Any opinions, findings, and conclusions or recommendations expressed in this material are those of the authors and do not necessarily reflect the views of the NSF.

\section{References}

1. Naghavi, M., Libby, P., Falk, E., Casscells, S., Litovsky, S., et al.: From vulnerable plaque to vulnerable patient: A call for new definitions and risk assessment strategies: Parts I-II. Circulation 108 (2003) 1664-1672/1772-1778

2. Kolodgie, F., Gold, H., Burke, A., Fowler, D., et al.: Intraplaque hemorrhage and progression of coronary atheroma. New England J of Med 349 (2003) 2316-2325

3. Hayden, M., Tyagi, S.: Vasa vasorum in plaque angiogenesis, metabolic syndrome, type 2 diabetes mellitus, and atheroscleropathy: a malignant transformation. Cardiovasc Diabetol 3 (2004)

4. Tearney, G., Yabushita, H., Houser, S., Aretz, H., Jang, I.K., et al.: Quantification of macrophage content in atherosclerotic plaques by optical coherence tomography. Circulation 107 (2003) 113-119

5. Khan, T., Soller, B., Naghavi, M., Casscells, W.: Tissue pH determination for the detection of metabolically active, inflamed vulnerable plaques using near-infrared spectroscopy: An in-vitro feasibility study. Cardiology 103 (2005) 10-16 
6. Go, V., Bhatt, M., Hendel, R.: The diagnostic and prognostic value of ECG-gated SPECT myocardial perfusion imaging. J Nucl Med 45 (2004) 912-921

7. Winter, P., Morawski, A., Caruthers, S., Fuhrhop, R., Zhang, H., et al:: Molecular imaging of angiogenesis in early-stage atherosclerosis with $\alpha_{v} \beta_{3}$-integrin-targeted nanoparticles. Circulation 108 (2003) 2270-2274

8. Olszewski, M., Wahle, A., Vigmostad, S., Sonka, M.: Multidimensional segmentation of coronary intravascular ultrasound images using knowledge-based methods. In: Proc SPIE Medical Imaging. Volume 5747. (2005) 496-504

9. Gossl, M., Rosol, M., Malyar, N., Fitzpatrick, L., Beighley, P., Zamir, M., Ritman, E.: Functional anatomy and hemodynamic characteristics of vasa vasorum in the walls of porcine coronary arteries. Anat Rec 272A (2003) 526-537

10. Zhu, H., Oakeson, K., Friedman, M.: Retrieval of cardiac phase from IVUS sequences. In: Proc SPIE Medical Imaging. Volume 5035. (2003) 135-146

11. Bruzzone, L., Prieto, D.: Automatic analysis of the difference image for unsupervised change detection. IEEE Trans Geo Remote Sensing 38 (2000) 1171-1182

12. Carlier, S., Kakadiaris, I.A., Dib, N., Vavuranakis, M., Stefanadis, C., O'Malley, S.M., et al.: Vasa vasorum imaging: A new window to the clinical detection of vulnerable atherosclerotic plaques. Curr Athero Rep 7 (2005) 164-169 